\title{
Figure S1.
}

Evolution of pigments over time of the two oil samples over 5 different storage conditions. A, chlorophylls in the Tunisian sample; B, chlorophylls in the Italian sample; C, carotenoids in the Tunisian sample; D, carotenoids in the Italian sample. The content of chlorophylls was evaluated by the formula " $\mathrm{mg}_{\mathrm{ph}} / \mathrm{kg}=$ $345.3^{*}\left(\lambda_{670}-\left(0.5^{*} \lambda_{630}\right)-\left(0.5^{*} \lambda_{710}\right)\right) / \mathrm{L}$ " and was expressed as $\mathrm{mg}_{\mathrm{ph}} / \mathrm{kg}$ (mg pheophytin-a per $\mathrm{kg}$ of oil). The evolution of the carotenoids was followed using the sum of the absorbance at $\lambda=460 \mathrm{~nm}+\lambda=490 \mathrm{~nm}$. All the measurements are the mean of three determinations. For each series of data, different letters over the bars indicate significant differences at $\mathrm{p}<0.05$
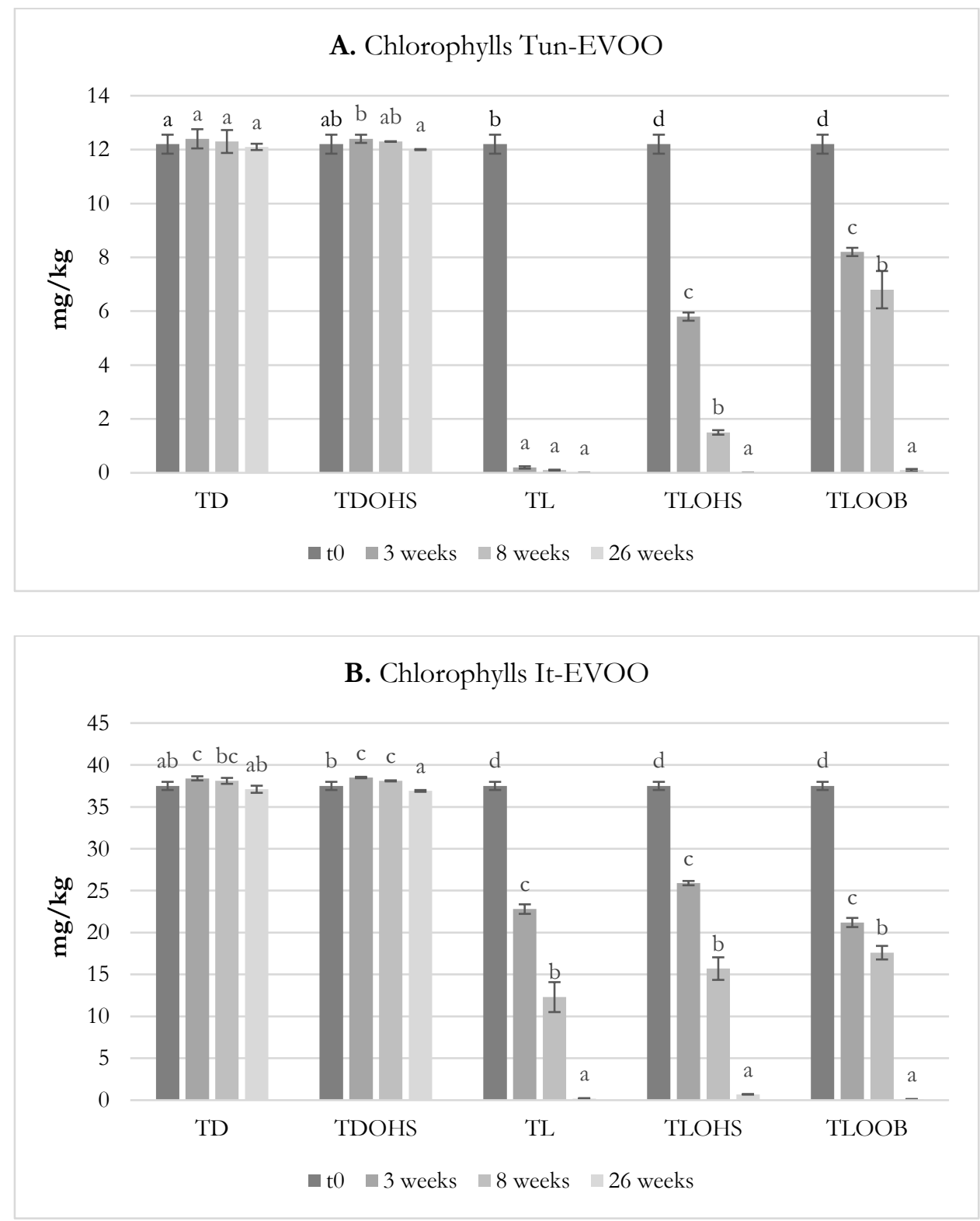

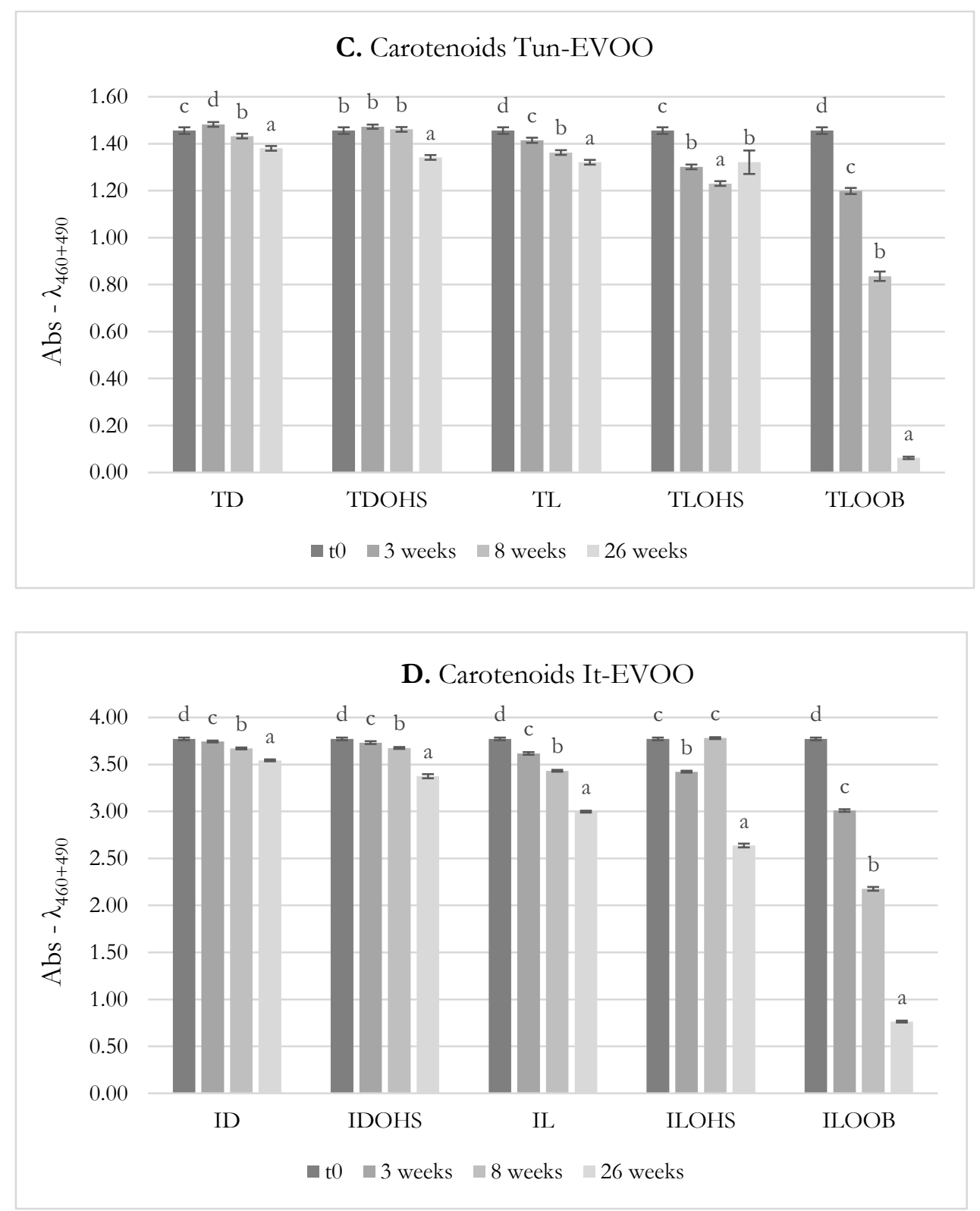
Figure S2. Appearance of the oil samples after 6 months of storage.

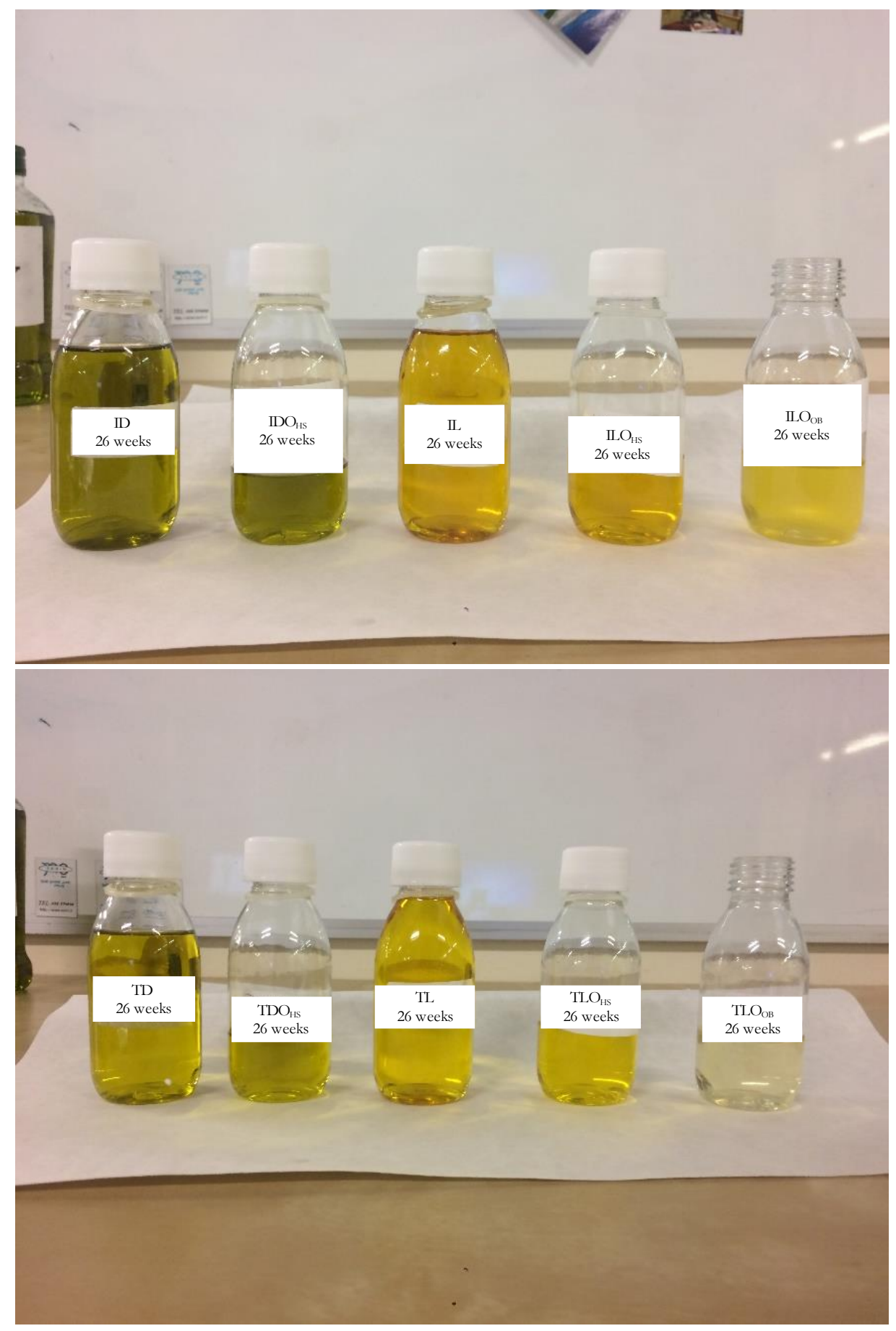


Table S1. List of the used authentic standards for analysis of Volatile Organic Compounds. The table also reports their purity and the selected internal standard used for quantitation for each VOC. All the standards are from Sigma Aldrich, Steinheim, Germany.

\begin{tabular}{|c|c|c|}
\hline Volatile Compound & $\begin{array}{c}\text { Purity } \\
(\%)\end{array}$ & Internal Standard \\
\hline heptane & $\geq 99.8$ & 1-butanol-d $\mathrm{d}_{10}$ \\
\hline octane & $\geq 99.7$ & toluene- $\mathrm{d}_{8}$ \\
\hline methyl acetate & $\geq 99.9$ & ethyl acetate- $\mathrm{d}_{8}$ \\
\hline ethyl acetate & $\geq 99.9$ & ethyl acetate- $\mathrm{d}_{8}$ \\
\hline methanol & $\geq 99.9$ & 4-methyl-2-pentanol \\
\hline butan-2-one & $\geq 99.9$ & trimethylacetaldehyde \\
\hline methyl propanoate & $\geq 99.9$ & trimethylacetaldehyde \\
\hline 2-methyl butanal & $\geq 95$ & trimethylacetaldehyde \\
\hline isovaleraldehyde & $\geq 97$ & trimethylacetaldehyde \\
\hline ethanol & 96 & 4-methyl-2-pentanol \\
\hline ethyl propanoate & $\geq 99.7$ & ethyl acetate- $\mathrm{d}_{8}$ \\
\hline pentan-3-one & 100 & ethyl acetate- $\mathrm{d}_{8}$ \\
\hline pentanal & $\geq 97.5$ & ethyl acetate- $\mathrm{d}_{8}$ \\
\hline butan-2-ol & $\geq 99.8$ & 1-butanol-d $\mathrm{d}_{10}$ \\
\hline pent-1-en-3-one & $\geq 97$ & 4-methyl-2-pentanol \\
\hline propanol & 99 & 1-butanol-d 10 \\
\hline ethyl butanoate & $\geq 99.5$ & 4-methyl-2-pentanol \\
\hline buthyl acetate & $\geq 99.9$ & 4-methyl-2-pentanol \\
\hline hexanal & $\geq 95$ & 4-methyl-2-pentanol \\
\hline isobutanol & $\geq 99.8$ & 1-butanol-d $\mathrm{d}_{10}$ \\
\hline pentan-2-ol & $\geq 97$ & 1-butanol-d $\mathrm{d}_{10}$ \\
\hline$E$-2-pentenal & $\geq 95$ & 4-methyl-2-pentanol \\
\hline pent-1-en-3-ol & $\geq 98$ & 1-butanol-d ${ }_{10}$ \\
\hline heptan-2-one & $\geq 99$ & 3-octanone \\
\hline heptanal & $\geq 97$ & 4-methyl-2-pentanol \\
\hline limonene & $\geq 99$ & 3-octanone \\
\hline 2-methylbutanol + & $\geq 98$ & 1-butanol-d \\
\hline 3-methylbutanol & $\geq 98.5$ & 1-Dutanol-d 10 \\
\hline$Z$-3-hexenal & $50 \%$ in triacetin & 4-methyl-2-pentanol \\
\hline E-2-hexenal & $\geq 97$ & 4-methyl-2-pentanol \\
\hline pentanol & $\geq 99.8$ & 4-methyl-2-pentanol \\
\hline hexyl acetate & $\geq 99.7$ & 6-chloro-2-hexanone \\
\hline octan-2-one & $\geq 99.5$ & 3-octanone \\
\hline octanal & $\geq 98$ & 3-octanone \\
\hline oct-1-en-3-one & $\geq 97$ & 3-octanone \\
\hline
\end{tabular}




\begin{tabular}{|c|c|c|}
\hline$E$-2-pentenol & 95 & 4-methyl-2-pentanol \\
\hline$Z$-2-pentenol & 95 & 4-methyl-2-pentanol \\
\hline heptan-2-ol & $\geq 98$ & 4-methyl-2-pentanol \\
\hline Z-3-hexenyl-acetate & $\geq 98$ & 6-chloro-2-hexanone \\
\hline$E$-2-hexenyl-acetate & 98 & 6-chloro-2-hexanone \\
\hline E-2-heptenal & $\geq 95$ & 3-octanone \\
\hline 6-methylhept-5-en-2-one & $\geq 97$ & 3-octanone \\
\hline hexanol & $\geq 99.9$ & 4-methyl-2-pentanol \\
\hline$E$-3-hexenol & 97 & 4-methyl-2-pentanol \\
\hline$Z$-3-hexenol & $\geq 98$ & 4-methyl-2-pentanol \\
\hline nonan-2-one & 95 & 3-octanone \\
\hline nonanal & $\geq 99.5$ & 3-octanone \\
\hline E-2-hexenol & 96 & 4-methyl-2-pentanol \\
\hline$Z$-2-hexenol & 95 & 4-methyl-2-pentanol \\
\hline$E, E$-hexa-2,4-dienal & $\geq 95$ & 3-octanone \\
\hline octan-2-ol & $\geq 97$ & 3-octanone \\
\hline$E$-2-octenal & $\geq 97$ & 3-octanone \\
\hline oct-1-en-3-ol & $\geq 98$ & 3-octanone \\
\hline heptanol & $\geq 99.9$ & 3-octanone \\
\hline acetic acid & $\geq 99$ & acetic acid-2,2,2-d3 \\
\hline decanal & $\geq 95$ & 3-octanone \\
\hline$E, E$-hepta-2,4-dienal & $\geq 88$ & 3-octanone \\
\hline benzaldehyde & $\geq 9.5$ & 3-octanone \\
\hline E-2-nonenal & $\geq 95$ & 3-octanone \\
\hline octanol & $\geq 99.7$ & 3-octanone \\
\hline propanoic acid & $\geq 99.8$ & 4-methyl-2-pentanol \\
\hline butanoic acid & $\geq 99.5$ & acetic acid-2,2,2- $\mathrm{d}_{3}$ \\
\hline$E$-2-decenal & $\geq 96$ & 3-octanone \\
\hline nonanol & 98 & 3-octanone \\
\hline$E, E$-nona-2,4-dienal & $\geq 90$ & 3-octanone \\
\hline pentanoic acid & $\geq 99.8$ & acetic acid-2,2,2-d3 \\
\hline$E, E$-deca-2,4-dienal & $\geq 84$ & 6-chloro-2-hexanone \\
\hline hexanoic acid & $\geq 99$ & acetic acid-2,2,2-d3 \\
\hline guaiacol & 99 & 3,4-dimethylphenol \\
\hline phenyl ethanol & 99 & 3,4-dimethylphenol \\
\hline Phenol & 99 & 3,4-dimethylphenol \\
\hline 4-ethylguaiacol & $\geq 98$ & 3,4-dimethylphenol \\
\hline 4-ethylphenol & 99.8 & 3,4-dimethylphenol \\
\hline
\end{tabular}


Table S2. List of the samples analyzed at time 0 and after 3, 8 and 26 weeks. I, Italy; T, Tunisia; D, dark; L, light; OHs, oxygen in headspace; Оoв, open bottle

\begin{tabular}{|c|c|c|c|}
\hline Sample & Code & $\begin{array}{c}\text { Light/Dark } \\
\text { exposure }\end{array}$ & Oxygen \\
\hline \multirow{5}{*}{ 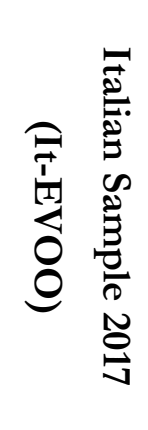 } & ID & Dark & No \\
\hline & $\mathrm{IDO}_{\mathrm{HS}}$ & Dark & Head Space \\
\hline & IL & Light & No \\
\hline & $\mathrm{ILO}_{\mathrm{HS}}$ & Light & Head Space \\
\hline & $\mathrm{ILO}_{\mathrm{OB}}$ & Light & Open Bottle \\
\hline \multirow{5}{*}{ 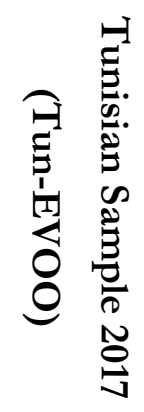 } & TD & Dark & No \\
\hline & $\mathrm{TDO}_{\mathrm{HS}}$ & Dark & Head Space \\
\hline & $\mathrm{TL}$ & Light & No \\
\hline & $\mathrm{TLO}_{\mathrm{HS}}$ & Light & Head Space \\
\hline & TLO $_{\text {Oв }}$ & Light & Open Bottle \\
\hline
\end{tabular}


Table S3. Peroxide values over time of the two extra virgin olive oils stored in several conditions. Data are expressed as meqo2/kg (mean \pm SD). In each raw, different letters indicate significant differences at $\mathrm{p}<0.05$.

I, Italian EVOO; T, Tunisian EVOO; D, Dark; O, Oxygen; L, Light; HS, HeadSpace; OB, Open Bottle

\begin{tabular}{|c|c|c|c|c|}
\hline & to & t1 - 3 weeks & t2 - 8 weeks & t3 - 26 weeks \\
\hline ID & $12.1 \pm 0.8 a b$ & $10.3 \pm 1.2 a$ & $14.3 \pm 1.1 c$ & $12.2 \pm 0.7 b$ \\
\hline $\mathrm{IDO}_{\mathrm{HS}}$ & $12.1 \pm 0.8 a$ & $10.1 \pm 1.7 a$ & $17.3 \pm 1.0 b$ & $19.1 \pm 1.2 b$ \\
\hline IL & $12.1 \pm 0.8 a$ & $12.3 \pm 0.9 a$ & $14.3 \pm 1.7 a$ & $14.7 \pm 3.9 a$ \\
\hline ILO $_{\mathrm{HS}}$ & $12.1 \pm 0.8 a$ & $42.8 \pm 1.5 b$ & $49.9 \pm 6.3 b c$ & $46.3 \pm 2.3 c$ \\
\hline \multirow[t]{2}{*}{$\mathrm{ILO}_{\mathrm{OB}}$} & $12.1 \pm 0.8 a$ & $89.0 \pm 0.3 b$ & $181.1 \pm 2.2 c$ & $461.8 \pm 16.5 d$ \\
\hline & t0 & t1 - 3 weeks & $\mathrm{t} 2-8$ weeks & t3 - 26 weeks \\
\hline TD & $14.0 \pm 1.1 a$ & $12.7 \pm 1.0 a$ & $18.1 \pm 0.7 b$ & $13.4 \pm 1.2 a$ \\
\hline $\mathrm{TDO}_{\mathrm{HS}}$ & $14.0 \pm 1.1 a$ & $14.5 \pm 1.7 a$ & $23.9 \pm 6.2 b$ & $21.5 \pm 1.0 b$ \\
\hline TL & $14.0 \pm 1.1 a$ & $15.7 \pm 1.5 a$ & $18.0 \pm 4.9 a$ & $16.5 \pm 2.1 a$ \\
\hline $\mathrm{TLO}_{\mathrm{HS}}$ & $14.0 \pm 1.1 a$ & $41.9 \pm 3.4 b$ & $52.7 \pm 1.9 d$ & $47.6 \pm 3.4 c$ \\
\hline $\mathrm{TLO}_{\mathrm{OB}}$ & $14.0 \pm 1.1 a$ & $79.0 \pm 1.1 b$ & $177.1 \pm 17.8 c$ & $478.5 \pm 19.9 d$ \\
\hline
\end{tabular}




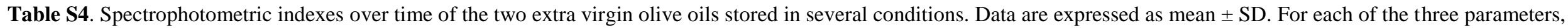

different letters in each raw indicate significant differences at $\mathrm{p}<0.05$

I, Italian EVOO; T, Tunisian EVOO; D, Dark; O, Oxygen; L, Light; HS, HeadSpace; OB, Open Bottle

\begin{tabular}{|c|c|c|c|c|c|c|c|c|c|c|c|c|}
\hline & \multicolumn{4}{|c|}{$\mathbf{K}_{232}$} & \multicolumn{4}{|c|}{$\mathrm{K}_{268}$} & \multicolumn{4}{|c|}{$\Delta \mathbf{K}$} \\
\hline & to & $\begin{array}{c}\mathrm{t} 1 \\
3 \text { weeks }\end{array}$ & $\begin{array}{c}\text { t2 } \\
\text { 8 weeks }\end{array}$ & $\begin{array}{c}\text { t3 } \\
26 \text { weeks } \\
\end{array}$ & to & $\begin{array}{c}\mathrm{t} 1 \\
3 \text { weeks }\end{array}$ & $\begin{array}{c}\text { t2 } \\
8 \text { weeks }\end{array}$ & $\begin{array}{c}t 3 \\
26 \text { weeks }\end{array}$ & to & $\begin{array}{c}\mathrm{t} 1 \\
3 \text { weeks }\end{array}$ & $\begin{array}{c}\mathrm{t} 2 \\
8 \text { weeks }\end{array}$ & $\begin{array}{c}\mathrm{t} 3 \\
26 \text { weeks }\end{array}$ \\
\hline ID & $2.04 \pm 0.08 a$ & $1.95 \pm 0.11 a$ & $1.96 \pm 0.09 a$ & $2.32 \pm 0.10 b$ & $0.15 \pm 0.01 a$ & $0.14 \pm 0.01 a$ & $0.14 \pm 0.01 a$ & $0.18 \pm 0.01 b$ & $-0.002 \pm 0.000 a$ & $-0.002 \pm 0.001 a$ & $-0.002 \pm 0.001 a$ & $-0.002 \pm 0.001 a$ \\
\hline $\mathrm{IDO}_{\mathrm{HS}}$ & $2.04 \pm 0.08 a$ & $2.01 \pm 0.23 a$ & $1.93 \pm 0.11 a$ & $2.57 \pm 0.18 b$ & $0.15 \pm 0.01 a b$ & $0.15 \pm 0.02 a b$ & $0.14 \pm 0.01 a$ & $0.16 \pm 0.01 b$ & $-0.002 \pm 0.000 a$ & $-0.002 \pm 0.001 a$ & $-0.001 \pm 0.001 a$ & $-0.002 \pm 0.001 a$ \\
\hline IL & $2.04 \pm 0.08 a b$ & $2.15 \pm 0.09 b c$ & $1.97 \pm 0.12 a$ & $2.23 \pm 0.03 c$ & $0.15 \pm 0.01 a$ & $0.19 \pm 0.01 b$ & $0.20 \pm 0.01 b$ & $0.27 \pm 0.01 c$ & $-0.002 \pm 0.000 a$ & $0.004 \pm 0.001 b$ & $0.007 \pm 0.001 c$ & $0.015 \pm 0.001 d$ \\
\hline $\mathrm{ILO}_{\mathrm{Hs}}$ & $2.04 \pm 0.08 a$ & $2.40 \pm 0.10 b$ & $2.07 \pm 0.02 a$ & $2.57 \pm 0.02 c$ & $0.15 \pm 0.01 a$ & $0.20 \pm 0.01 b$ & $0.19 \pm 0.01 b$ & $0.29 \pm 0.01 c$ & $-0.002 \pm 0.000 a$ & $0.003 \pm 0.001 b$ & $0.007 \pm 0.001 c$ & $0.017 \pm 0.001 d$ \\
\hline \multirow[t]{2}{*}{$\mathrm{ILO}_{\mathrm{oB}}$} & $2.04 \pm 0.08 a$ & $2.56 \pm 0.19 b$ & $3.04 \pm 0.15 c$ & $6.11 \pm 0.34 d$ & $0.15 \pm 0.01 a$ & $0.20 \pm 0.01 a$ & $0.21 \pm 0.01 a$ & $0.59 \pm 0.08 b$ & $-0.002 \pm 0.000 a$ & $0.002 \pm 0.000 b$ & $0.003 \pm 0.001 b c$ & $0.004 \pm 0.001 c$ \\
\hline & to & $\begin{array}{c}\mathrm{t} 1 \\
3 \text { weeks }\end{array}$ & $\begin{array}{c}\mathrm{t} 2 \\
8 \text { weeks }\end{array}$ & $\begin{array}{c}\mathrm{t} 3 \\
26 \text { weeks }\end{array}$ & to & $\begin{array}{c}\mathrm{t} 1 \\
3 \text { weeks }\end{array}$ & $\begin{array}{c}\mathrm{t} 2 \\
8 \text { weeks }\end{array}$ & $\begin{array}{c}\mathrm{t} 3 \\
26 \text { weeks }\end{array}$ & to & $\begin{array}{c}\mathrm{t} 1 \\
\text { 3 weeks }\end{array}$ & $\begin{array}{c}\mathrm{t} 2 \\
8 \text { weeks }\end{array}$ & $\begin{array}{c}\mathrm{t} 3 \\
26 \text { weeks }\end{array}$ \\
\hline TD & $2.02 \pm 0.07 a$ & $1.93 \pm 0.07 a$ & $2.03 \pm 0.09 a$ & $1.98 \pm 0.10 a$ & $0.14 \pm 0.01 a$ & $0.15 \pm 0.01 a$ & $0.15 \pm 0.01 a$ & $0.15 \pm 0.01 a$ & $0.003 \pm 0.000 a$ & $0.003 \pm 0.001 a$ & $0.002 \pm 0.001 a$ & $0.002 \pm 0.001 a$ \\
\hline TDO $_{\text {Hs }}$ & $2.02 \pm 0.07 a$ & $2.02 \pm 0.03 a$ & $1.95 \pm 0.15 a$ & $2.80 \pm 0.07 b$ & $0.14 \pm 0.01 a$ & $0.16 \pm 0.01 b c$ & $0.15 \pm 0.01 a b$ & $0.17 \pm 0.01 c$ & $0.003 \pm 0.000 c$ & $0.002 \pm 0.001 b$ & $0.001 \pm 0.001 a$ & $0.001 \pm 0.001 a$ \\
\hline TL & $2.02 \pm 0.07 a$ & $2.10 \pm 0.10 a b$ & * & $2.25 \pm 0.16 b$ & $0.14 \pm 0.01 a$ & $0.19 \pm 0.01 b$ & $0.15 \pm 0.02 a$ & $0.22 \pm 0.02 c$ & $0.003 \pm 0.000 a$ & $0.007 \pm 0.001 b$ & $0.007 \pm 0.001 b$ & $0.012 \pm 0.001 c$ \\
\hline TLO $_{\mathrm{HS}}$ & $2.02 \pm 0.07 a$ & $2.57 \pm 0.15 b$ & $2.65 \pm 0.42 b c$ & $3.09 \pm 0.15 c$ & $0.14 \pm 0.01 a$ & $0.23 \pm 0.01 b$ & $0.25 \pm 0.05 b$ & $0.32 \pm 0.01 c$ & $0.003 \pm 0.000 a$ & $0.008 \pm 0.001 b$ & $0.012 \pm 0.002 c$ & $0.018 \pm 0.001 d$ \\
\hline TLOов & $2.02 \pm 0.07 a$ & $3.31 \pm 0.20 a b$ & $4.03 \pm 0.39 b$ & $8.21 \pm 1.62 c$ & $0.14 \pm 0.01 a$ & $0.25 \pm 0.02 b$ & $0.23 \pm 0.02 b$ & $0.45 \pm 0.03 c$ & $0.003 \pm 0.000 a$ & $0.007 \pm 0.001 c$ & $0.007 \pm 0.001 c$ & $0.005 \pm 0.001 b$ \\
\hline
\end{tabular}


Table S5. Values of absorption at the $\lambda$ selected for evaluating pigments in the analyzed oils. $\lambda_{460}$ and $\lambda_{490}$ were selected for carotenoids according to figure $\mathrm{S} 1$ and the evolution of the carotenoid was followed using the sum of these wavenumbers $\left(\lambda_{460}+\lambda_{460}\right)$. The content of chlorophylls was evaluated by the formula " $\mathrm{mg}_{\text {chlor }} / \mathrm{kg}=$ $345.3^{*}\left(\lambda_{670}\left(0.5^{*} \lambda_{630}\right)-\left(0.5^{*} \lambda_{710}\right)\right)$ " and was expressed as $\mathrm{mg}_{\text {chlor }} / \mathrm{kg}$. The evolution of the carotenoids content was evaluated as the sum of the absorbance at $\lambda=460 \mathrm{~nm}+\lambda=490 \mathrm{~nm}$. All the measurements are the mean of three determinations

\begin{tabular}{|c|c|c|c|c|c|c|c|}
\hline \multicolumn{8}{|c|}{ It-EVOO } \\
\hline & $\lambda_{460}$ & $\lambda_{490}$ & $\lambda_{460}+\lambda_{460}$ & $\lambda_{630}$ & $\lambda_{670}$ & $\lambda_{710}$ & $\begin{array}{c}\mathbf{m g}_{\mathrm{ph}} / \mathrm{Kg} \\
\text { oil }\end{array}$ \\
\hline ITALY_T0 & 2.1642 & 1.6080 & 3.7722 & 0.0695 & 1.1122 & -0.0170 & 37.5 \\
\hline ID_T1 & 2.1431 & 1.6014 & 3.7445 & 0.0614 & 1.1295 & -0.0240 & 38.4 \\
\hline ID_T2 & 2.1069 & 1.5639 & 3.6708 & 0.0628 & 1.1237 & -0.0200 & 38.1 \\
\hline ID_T3 & 2.0358 & 1.5080 & 3.5438 & 0.0606 & 1.0949 & -0.0180 & 37.1 \\
\hline ITALY_T0 & 2.1642 & 1.6080 & 3.7722 & 0.0695 & 1.1122 & -0.0170 & 37.5 \\
\hline IDOHs_T1 & 2.1369 & 1.5959 & 3.7328 & 0.0610 & 1.1345 & -0.0240 & 38.5 \\
\hline IDOHS_T3 & 2.1011 & 1.5747 & 3.6757 & 0.0606 & 1.1224 & -0.0210 & 38.1 \\
\hline IDOнs_T3 & 1.9385 & 1.4366 & 3.3751 & 0.0593 & 1.0896 & -0.0157 & 36.9 \\
\hline ITALY_T0 & 2.1642 & 1.6080 & 3.7722 & 0.0695 & 1.1122 & -0.0170 & 37.5 \\
\hline IL_T1 & 2.0545 & 1.5628 & 3.6173 & 0.0453 & 0.6703 & -0.0230 & 22.8 \\
\hline IL_T2 & 1.9611 & 1.4717 & 3.4328 & 0.0243 & 0.3545 & -0.0253 & 12.3 \\
\hline IL_T3 & 1.7386 & 1.2595 & 2.9980 & -0.0170 & -0.0160 & -0.0280 & 0.2 \\
\hline ITALY_T0 & 2.1642 & 1.6080 & 3.7722 & 0.0695 & 1.1122 & -0.0170 & 37.5 \\
\hline ILOHs_T1 & 1.9881 & 1.4352 & 3.4233 & 0.0477 & 0.7657 & -0.0190 & 25.9 \\
\hline ILOHs_T2 & 1.9261 & 1.8543 & 3.7804 & 0.0248 & 0.4555 & -0.0237 & 15.7 \\
\hline ILOHs_T3 & 1.5683 & 1.0692 & 2.6374 & -0.0197 & -0.0037 & -0.0260 & 0.6 \\
\hline ITALY_T0 & 2.1642 & 1.6080 & 3.7722 & 0.0695 & 1.1122 & -0.0170 & 37.5 \\
\hline ILOовT1 & 1.7888 & 1.2208 & 3.0096 & 0.0471 & 0.6312 & -0.0120 & 21.2 \\
\hline ILOовT2 & 1.3495 & 0.8267 & 2.1762 & 0.0324 & 0.5181 & -0.0167 & 17.6 \\
\hline ILOовT3 & 0.4448 & 0.3187 & 0.7635 & 0.1112 & 0.1017 & 0.0853 & 0.1 \\
\hline
\end{tabular}




\begin{tabular}{|c|c|c|c|c|c|c|c|}
\hline \multicolumn{8}{|c|}{ Tun-EVOO } \\
\hline & $\lambda_{460}$ & $\lambda_{490}$ & $\lambda_{460}+\lambda_{460}$ & $\lambda_{630}$ & $\lambda_{670}$ & $\lambda_{710}$ & $\begin{array}{c}\mathrm{mg}_{\mathrm{ph}} / \mathrm{Kg} \\
\text { oil }\end{array}$ \\
\hline TUNISIA_T0 & 0.8427 & 0.6132 & 1.4559 & -0.0010 & 0.3368 & -0.0310 & 12.2 \\
\hline TD1 & 0.8562 & 0.6259 & 1.4821 & -0.0040 & 0.3416 & -0.0330 & 12.4 \\
\hline TD2 & 0.8323 & 0.6003 & 1.4326 & -0.0070 & 0.3357 & -0.0350 & 12.3 \\
\hline TD3 & 0.8070 & 0.5732 & 1.3802 & -0.0070 & 0.3294 & -0.0350 & 12.1 \\
\hline TUNISIA_T0 & 0.8427 & 0.6132 & 1.4559 & -0.0010 & 0.3368 & -0.0310 & 12.2 \\
\hline TDOHs1 & 0.8521 & 0.6204 & 1.4725 & -0.0050 & 0.3409 & -0.0340 & 12.4 \\
\hline TDOHS 2 & 0.8463 & 0.6147 & 1.4610 & -0.0037 & 0.3395 & -0.0323 & 12.3 \\
\hline TDOHs3 & 0.7794 & 0.5622 & 1.3416 & -0.0047 & 0.3292 & -0.0323 & 12.0 \\
\hline TUNISIA_T0 & 0.8427 & 0.6132 & 1.4559 & -0.0010 & 0.3368 & -0.0310 & 12.2 \\
\hline TL1 & 0.8169 & 0.5977 & 1.4146 & -0.0230 & -0.0210 & -0.0330 & 0.2 \\
\hline TL2 & 0.7892 & 0.5733 & 1.3626 & -0.0290 & -0.0297 & -0.0343 & 0.1 \\
\hline TL3 & 0.7655 & 0.5557 & 1.3212 & -0.0037 & -0.0055 & -0.0054 & nd \\
\hline TUNISIA_T0 & 0.8427 & 0.6132 & 1.4559 & -0.0010 & 0.3368 & -0.0310 & 12.2 \\
\hline TLOHS1 1 & 0.7644 & 0.5370 & 1.3014 & -0.0140 & 0.1430 & -0.0330 & 5.8 \\
\hline TLOHS2 & 0.7279 & 0.5027 & 1.2306 & -0.0257 & 0.0142 & -0.0340 & 1.5 \\
\hline TLOHs 3 & 0.7655 & 0.5557 & 1.3212 & -0.0317 & -0.0320 & -0.0327 & nd \\
\hline TUNISIA_T0 & 0.8427 & 0.6132 & 1.4559 & -0.0010 & 0.3368 & -0.0310 & 12.2 \\
\hline TLOов1 & 0.7171 & 0.4816 & 1.1987 & -0.0060 & 0.2210 & -0.0290 & 8.2 \\
\hline TLOов2 & 0.5221 & 0.3136 & 0.8357 & -0.0123 & 0.1766 & -0.0307 & 6.8 \\
\hline ТLОов3 & 0.0434 & 0.0190 & 0.0624 & -0.0237 & -0.0237 & -0.0270 & 0.1 \\
\hline
\end{tabular}


Table S6. Fatty acid precursors of the main VOCs produced during oxidation processes, according to previous literature. $O$, oleic acid; L, linoleic acid; Ln, linolenic acid

\begin{tabular}{|c|c|c|c|c|}
\hline VOC & $\begin{array}{c}\text { Morales et } \\
\text { al., } 1997\end{array}$ & $\begin{array}{c}\text { Choe \& Min } \\
2006\end{array}$ & $\begin{array}{c}\text { Luna et al., } \\
2006\end{array}$ & $\begin{array}{c}\text { Frankel } \\
2010\end{array}$ \\
\hline Propanal & & Ln & $\mathrm{Ln}$ & \\
\hline Butanal & & $\mathrm{Ln}$ & & \\
\hline Pentanal & $\mathrm{L}$ & $\mathrm{L}$ & $\mathrm{L}$ & \\
\hline Hexanal & $\mathrm{L}$ & $\mathrm{L}$ & $\mathrm{L}$ & $\mathrm{L}$ \\
\hline Heptanal & $\mathrm{L}$ & & $\mathrm{O}$ & \\
\hline Octanal & $\mathrm{O}$ & $\mathrm{O}$ & $\mathrm{O}$ & $\mathrm{O}$ \\
\hline Nonanal & $\mathrm{O}$ & $\mathrm{O}$ & $\mathrm{O}$ & \\
\hline Decanal & $\mathrm{O}$ & $\mathrm{O}$ & & \\
\hline$E$-2-butenal & & Ln & $\mathrm{Ln}$ & \\
\hline$E$-2-pentenal & & $\mathrm{Ln}$ & $\mathrm{Ln}$ & \\
\hline Z-3-hexenal & & & & Ln \\
\hline$E$-2-hexenal & Ln & Ln & & Ln \\
\hline$E$-2-heptenal & $\mathrm{L}$ & & $\mathrm{L}$ & \\
\hline$E$-2-octenal & $\mathrm{L}$ & $\mathrm{L}$ & $\mathrm{L}$ & \\
\hline Z-2-nonenal & & & & $\mathrm{L}$ \\
\hline$E$-2-nonenal & $\mathrm{L}$ & $\mathrm{L}$ & & $\mathrm{L}$ \\
\hline$E$-2-decenal & $\mathrm{O}$ & $\mathrm{O}$ & $\mathrm{O}$ & \\
\hline Hepta-2,4-dienal & Ln & & & \\
\hline Nona-2,4-dienal & & & & Ln \\
\hline Deca-2,4-dienal & $\mathrm{L}$ & $\mathrm{L}$ & & $\mathrm{L}$ \\
\hline Pentane & & $\mathrm{L} / \mathrm{Ln}$ & & \\
\hline Heptane & & $\mathrm{O}$ & & \\
\hline Octane & & $\mathrm{O}$ & $\mathrm{O}$ & \\
\hline Heptan-1-ol & & $\mathrm{O}$ & & \\
\hline Pentan-1-ol & & $\mathrm{L}$ & & \\
\hline Oct-1-en-3-ol & & $\mathrm{L}$ & & \\
\hline
\end{tabular}

